\title{
Wrist and Arm Body Surface Bipolar ECG Leads Signal and Sensor Study for Long-term Rhythm Monitoring
}

\author{
Omar J Escalona ${ }^{1}$, Louise McFrederick ${ }^{1}$, Maira Borges ${ }^{2}$, Pedro Linares ${ }^{2}$, Ricardo Villegas ${ }^{2}$, \\ Gilberto I Perpiñan ${ }^{3}$, James McLaughlin ${ }^{1}$, David McEneaney ${ }^{4}$ \\ ${ }^{1}$ University of Ulster, Newtownabbey, Northern Ireland \\ ${ }^{2}$ Universidad de Carabobo, Valencia, Venezuela. ${ }^{3}$ Universidad Simón Bolívar, Caracas, Venezuela \\ ${ }^{4}$ Craigavon Area Hospital, Portadown, Northern Ireland
}

\begin{abstract}
With cardiovascular disease and heart arrhythmias continuing to have a high mortality rate, it is important to monitor the electrocardiogram (ECG) signal in a noninvasive, long-term wearable device. In this study we investigate sensors and the ECG signal-to-noise ratio map along the left arm, for wearable arm-ECG monitoring devices. In a pilot study, 11 subjects attending a cardiology outpatient clinic, far-field left-arm ECG recordings included signals from a combination of dry and special pre-gelled BIS-Quatro ${ }^{T M}$ sensor system, axially and transversally oriented along the left arm: on the wrist, upper forearm and upper arm. A total of 10 bipolar leads were recorded simultaneously (using 18 acquisition channels). Each subject was recorded for 8 minutes at rest, using the bio-potential acquisition system; all data was imported and processed using Matlab and MS Excel. Analysis was completed to evaluate signal-to-noise ratio (SNR) distribution maps. An average ECG SNR figure of 42.63 was found in the dry-electrode positioned on the upper arm bipolar lead, whilst the SNR ratio positioned on the wrist was 13.14. Similar to this, in the BIS-electrodes (gelled), there was an average ECG SNR figure of 89.25 on the upper arm and of 5.18 positioned on the wrist. This study clinically evidenced the ECG S/N map on the left arm. It reveals that bipolar arm-ECG SNR are consistently stronger on the upper arm, when recorded with the gelled BIS sensors.
\end{abstract}

\section{Introduction}

Cardiovascular disease is the single most common cause of death in the general population: $50 \%$ of these are caused by abnormal heart patterns (arrhythmias). Atrial fibrillation (the most common arrhythmia) is associated with increased risk of stroke and heart failure in the aged population [1]. Many patients may not be aware that they have atrial fibrillation as it is not associated with any symptoms. If arrhythmias are detected at an early stage of heart disease appropriate treatment can be effective, reducing disability and death. However, arrhythmias in the early stages of heart disease may be transient, lasting only a few seconds, and thus difficult to detect. Current approaches to monitoring of cardiac rhythm fall into two categories. Event recorders are external recording devices attached by disposable self-adhesive electrodes to the patient's chest wall (figure 1a); they are inconvenient for the patient and only suitable for short term recording. Implantable loop recorders are inserted beneath the skin of the chest wall (figure 1b). While they are capable of monitoring heart rhythm for extended periods there is, considerable expense associated with the device and hospitalisation costs, and the risks of surgery including infection [2]. Monitoring the human physiological state on a quasi-permanent basis has become on increasing demand. Currently available cardiac monitoring devices do provide aid in monitoring the heart rate; these cannot reveal electrocardiographic features of the heart rhythm [3]. With cardiovascular disease and heart arrhythmias continuing to have a high mortality rate, it is important to monitor the electrocardiogram (ECG) signal in a noninvasive, long-term wearable device [4]. The aim of this study is to determine a suitable electrode and position along the left-arm for ECG far-field bipolar leads in long term rhythm monitoring devices.

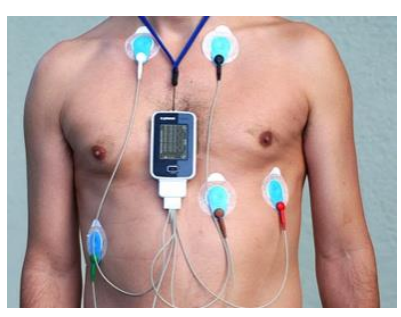

(a)

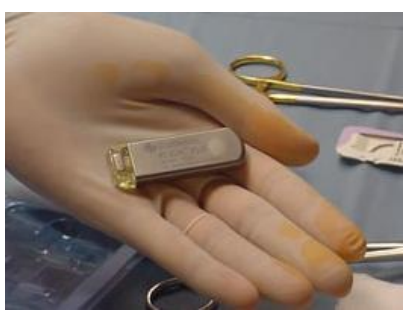

(b)
Figure 1. (a) Traditional Holter Monitor. (b) Implantable loop recorder. 


\section{Methods}

In order to establish a method for the recovery of ECG rhythm information from far field sites, such as the wrist, a study of the ECG signal levels and their prevalence over the background noise level was characterised after the application of conventional ECG denoising techniques. The study maps the gradient of signal degradation as distance from the heart increases along the extremity of the subject's left limb. A conventional high-resolution ECG signal averaging technique as described in [5] was used for the ECG denoising process; as it is a widely accepted method for small cardiac QRS synchronised potential recovery.

\subsection{Clinical ECG recordings}

Ethical approval for the investigation was obtained from the Office of Research Ethical Committees Northern Ireland (ORECNI). An information sheet was provided and informed signed consent obtained. Each subject was connected to a BioSemi-II data recorder system using two types of electrodes; dry studs $\mathrm{AgCl}$ fixed on an elastic strap, as seen in figure 2 (Ringtrode, Klazienaveen), and special pre-gelled sensor system strap as seen in figure 3 (BIS-Quatro $^{\mathrm{TM}}$, Medtronic). The electrode positions correspond to the comfort zones established by Gemperle et al.[6]. For this pilot study stage, left arm sensor channels were anatomically positioned as depicted in figure 4, and bipolar arm-ECG leads as defined in table 1. BioSemi-II driven right leg (DRL) and common (CMS) electrodes were positioned as indicated in figure 4 .

The subjects were selected from the general intake of the cardiology department at Craigavon Area Hospital. The subjects (age range 42-78 years) Body mass index was recorded. Recordings were carried out on different days using the same room environment and recording equipment. A database of 11 recordings from the left arm was created and processed using Mathworks Matlab programming environment. Recording duration was 500 seconds at a sample rate of $2048 \mathrm{~Hz}$ and 24 bit resolution; this gives a measurement resolution of $31.25 \mathrm{e}-9 \mathrm{~V}$ per bit.

\subsection{Analysis}

Analysis of ECG study recordings was performed by using a MATLAB tool developed for this purpose. As shown in Figure 5, input data is imported and pre-filtered by the tool and fiducial points are extracted for each relevant QRS complex. Later, an average of selected leads is obtained by applying the Single Fiducial Point (SFP) alignment technique [7] on them and results of the whole process are exported to three different Excel files for SNR map evaluation. The main processing stages are described in the following sections.

\section{3. $\quad$ Pre-filtering}

In this stage, significant leads are extracted from 18 acquisition channels according to relevant locations of electrodes distributed on the left arm, as described in table 1. These signals are then pre-filtered by applying a Notch filter (centre frequency $50 \mathrm{~Hz}$, quality factor $\mathrm{Q}=12.5$ ) to eliminate potential power line interferences, and a bandpass filter $\left(4^{\text {th }}\right.$ order Butterworth high-pass and low-pass filters, $0.2 \mathrm{~Hz}$ and $40 \mathrm{~Hz}$ cut-off frequencies, respectively) to denoise and smooth the signals.

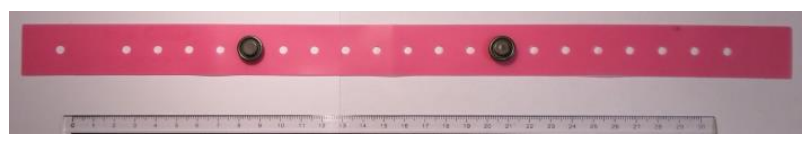

Figure 2. Dry electrodes ( $\mathrm{AgCl}$ studs) on elastic band.

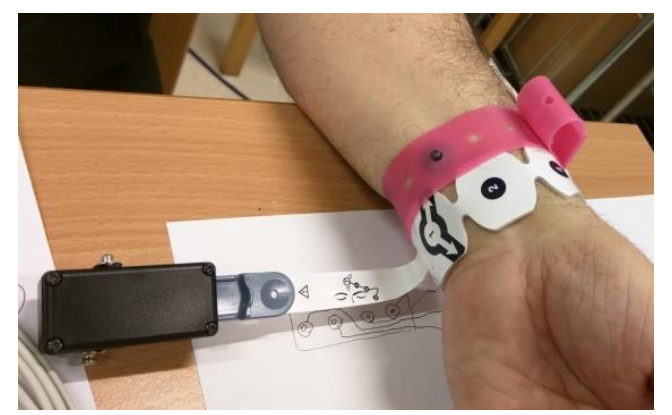

Figure 3. BIS-Quatro sensor strap with fixing dry electrodes elastic band on the wrist.

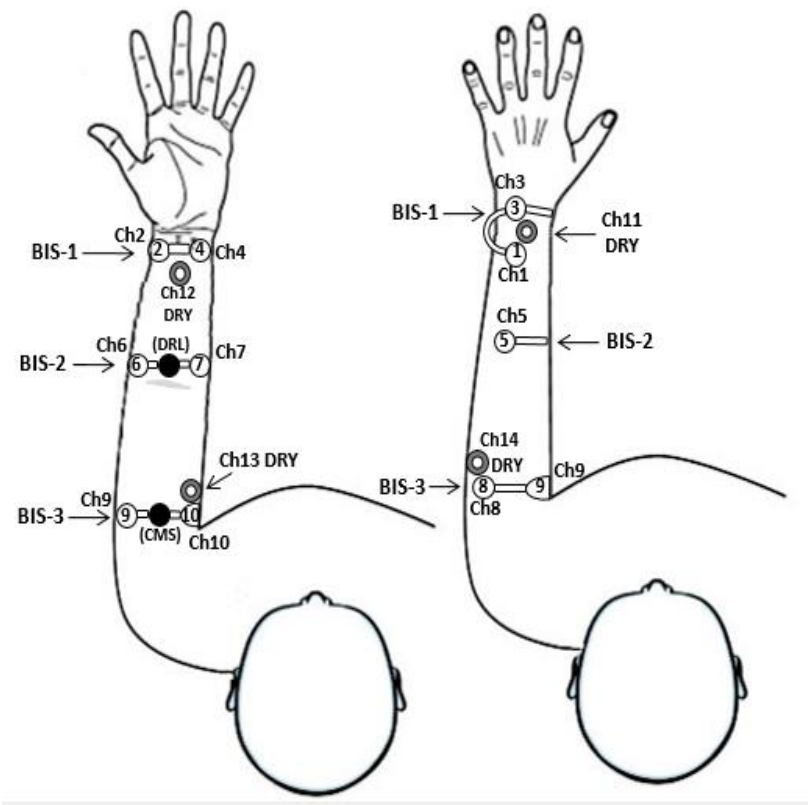

Figure 4. Anatomical Position of the left-arm bipolar leads, using the dry electrodes and 3 BIS-Quatro ${ }^{\mathrm{TM}}$ sensor strips. 


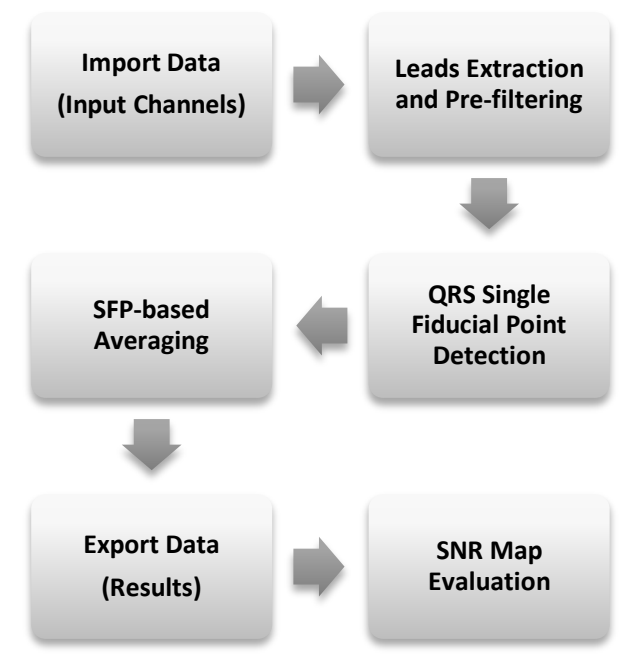

Figure 5. Process diagram for ECG signal analysis and SNR map evaluation

Table 1. Reference table of left-arm bipolar leads and anatomical locations.

\begin{tabular}{|c|c|c|c|}
\hline Lead & Channels & Location & $\begin{array}{c}\text { Electrode Type } \\
\text { (Orientation) }\end{array}$ \\
\hline 1 & Ch13-Ch14 & Upper arm & \multirow{2}{*}{$\begin{array}{c}\text { Dry } \\
\text { (Transversal) }\end{array}$} \\
\hline 2 & Ch11-Ch12 & Wrist & \\
\hline 3 & Ch13-Ch12 & Upper arm-Wrist & Dry (Axial) \\
\hline 4 & Ch10-Ch9 & Upper arm & \multirow{3}{*}{$\begin{array}{c}\text { Gelled } \\
\text { BIS-Quatro }^{\mathrm{TM}} \\
\text { (Transversal ) }^{\text {(T) }}\end{array}$} \\
\hline 5 & Ch7-Ch6 & Forearm & \\
\hline 6 & $\mathrm{Ch} 4-\mathrm{Ch} 2$ & Wrist & \\
\hline 7 & Ch10-Ch6 & Upper arm-Forearm & \multirow{4}{*}{$\begin{array}{c}\text { Gelled } \\
\text { BIS-Quatro }^{\mathrm{TM}} \\
\text { (Axial) }^{\text {A }}\end{array}$} \\
\hline 8 & $\mathrm{Ch} 7-\mathrm{Ch} 2$ & Forearm-Wrist & \\
\hline 9 & Ch10-Ch2 & Upper arm-Wrist & \\
\hline 10 & Ch1-Ch3 & Up wrist-Low wrist & \\
\hline Lead I & Limb leads & Chest (LA - RA) & Gelled (Standard) \\
\hline
\end{tabular}

\subsection{QRS fiducial points detection}

In the next processing stage, a time series of fiducial points is generated by tracking zero-level crossing in the RS interval of chest Lead I (selected as the reference channel), after being high-pass filtered at $2 \mathrm{~Hz}$ cut-off frequency, and low-pass filtered at $30 \mathrm{~Hz}$ cut-off frequency, both processes being $2^{\text {nd }}$ order Butterworth [7]. Then, by finding the maximum value $R_{\max }$ within a time window starting at $20 \mathrm{~s}$ and spanning for $50 \mathrm{~s}$, the algorithm then proceeds to search for signal values above a threshold $60 \%$ of $\mathrm{R}_{\max }$ followed by zero-level crossing (Figure 6); if the number of samples of the signal in this RS interval is contained within a valid time duration criteria of minimum time, $\mathrm{RDT}_{\mathrm{MIN}}=15$ samples time, and maximum, $\mathrm{RDT}_{\mathrm{MAX}}=50$ samples, the $\mathrm{QRS}$ complex is validated and its corresponding fiducial point is recorded.

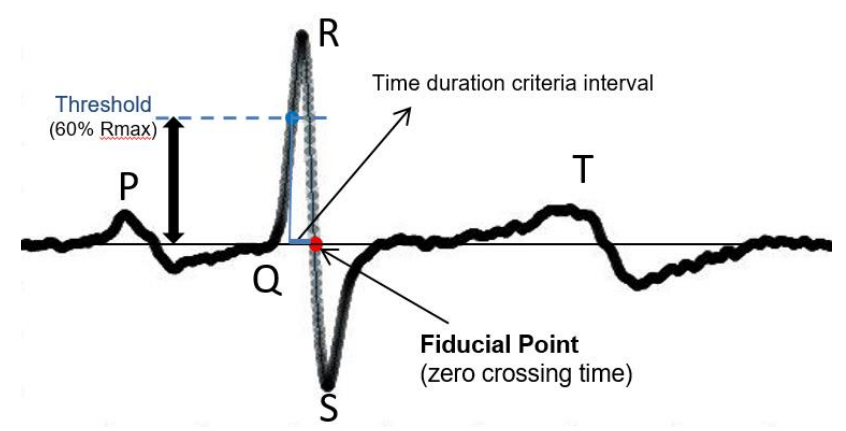

Figure 6. Parameters involved on the SFP QRS fiducial point detection algorithm, after ECG band-pass: 3-30 Hz.

\subsection{SFP signal averaging}

Signal averaged (SA) windows for the defined arm leads are calculated by applying the SFP alignment technique on the reference chest ECG Lead I. For doing the SA, the algorithm traverses each arm lead, 1400 sample values within time windows that span $300 \mathrm{~ms}$ to the left and $400 \mathrm{~ms}$ to the right of every fiducial point (Fig. 6), and accumulates the corresponding SA window samples for each incoming SFP time reference point. At the end, those partial accumulated values are divided by the number of fiducial points, as expressed in Eq. (1).

$$
\begin{aligned}
& A v g_{\text {eead }_{k}}=\frac{1}{N_{\text {fid }}}\left[\sum_{j=1}^{N_{\text {fid }}} \operatorname{Lead}_{k}\left(t_{s}^{j}\right), \cdots\right] \\
&, \forall s \in \text { sampling set of window } \\
&
\end{aligned}
$$

\subsection{SNR measurement}

Signal to noise ratio (SNR) for the defined leads computed SA were calculated by taking the peak-to-peak ECG QRS amplitude in a span of $120 \mathrm{~ms}$ (R-wave centred). Following this, a noise span of $40 \mathrm{~ms}$ positioned in the T-R time interval of the ECG in order to calculate the peak-to-peak noise amplitude. The Signal to noise ratio was then calculated as ECG amplitude/noise amplitude, which can also be expressed in the equation 2 .

$$
S N R=\frac{(Q R S) E C G_{p-p}}{(T-P) n o i s e_{p-p}}
$$

\section{Results}

As shown in Table 2, an average ECG Signal to Noise Ratio (SNR) of 34.90 was found in lead 1 using the dry $\mathrm{AgCl}$ electrodes; this was positioned on the upper arm. A high SRN (128.3) was also found in lead 4 which used 
Table 2. Average values ( $\mathrm{N}=11$ ) of measured ECG Signal (QRS complex), Noise Amplitude (in the T-P time segment) and Signal to Noise Ratio for Dry $\mathrm{AgCl}$ Electrodes and BIS (gelled) electrodes across Transversial and Axial lines.

\begin{tabular}{|c|c|c|c|c|c|c|c|c|c|c|c|}
\hline \multirow[b]{2}{*}{ Measurement } & \multicolumn{3}{|c|}{ Dry AgCl Electrodes } & \multicolumn{3}{|c|}{ Transversial BIS Electrodes } & \multicolumn{4}{|c|}{ Axial BIS Electrodes } & \multirow{2}{*}{$\frac{\text { Chest }}{\text { Lead I }}$} \\
\hline & $\begin{array}{c}\text { Lead- } \\
1\end{array}$ & $\begin{array}{l}\text { Lead- } \\
2\end{array}$ & $\begin{array}{c}\text { Lead- } \\
\mathbf{3}\end{array}$ & $\begin{array}{c}\text { Lead- } \\
4\end{array}$ & $\begin{array}{c}\text { Lead- } \\
5\end{array}$ & $\begin{array}{c}\text { Lead- } \\
6\end{array}$ & $\begin{array}{c}\text { Lead- } \\
7 \\
\end{array}$ & $\begin{array}{c}\text { Lead- } \\
8 \\
\end{array}$ & $\begin{array}{c}\text { Lead- } \\
9\end{array}$ & $\begin{array}{c}\text { Lead- } \\
10\end{array}$ & \\
\hline ECG Amplitude & 22.84 & 1.82 & 16.91 & 11.85 & 0.88 & 0.27 & 10.11 & 1.11 & 10.57 & 0.66 & 157.36 \\
\hline Noise Amplitude & 1.25 & 1.44 & 0.97 & 0.39 & 0.18 & 0.28 & 0.46 & 0.33 & 0.58 & 0.32 & 1.75 \\
\hline SNR & 34.90 & 2.14 & 36.13 & 128.3 & 8.27 & 1.70 & 59.39 & 5.16 & 41.84 & 3.39 & 181.93 \\
\hline
\end{tabular}

the $\mathrm{BIS}^{\mathrm{TM}}$ sensor electrodes positioned transversally on the upper arm. The axial bipolar lead 7, using BIS ${ }^{\mathrm{TM}}$ sensors on the upper arm and on the forearm, also presented a high SNR mean value of 59.39. Bipolar lead 6 , using the BIS ${ }^{\mathrm{TM}}$ sensor electrodes positioned transversally on the wrist, presented a significantly lower SNR of 1.70. Subsequently the bipolar lead 10 which was positioned axially along the wrist (between upper wrist and lower wrist) had a relatively better SNR of 3.39.

\section{Discussion and Conclusions}

The evidenced clinical information presented by the results in this paper shows a variance in the ability to measure detectable levels of heart originated electrogram signals at the extremities of the left arm. This in itself is promising. This pilot study has set out to investigate the cardiac electrogram signal strengths of bipolar ECG Lead sets, as their location and sensor type (dry or gelled) is changed along the left arm. Assuming a constant background noise amplitude (ECG p-p in T-P $\mathrm{P}_{40 \mathrm{~ms}}$ ) along the left-arm; from the upper-arm to the wrist, the mapped surface potential signal strength measurements (ECG p-p in $\mathrm{QRS}_{120 \mathrm{~ms}}$ ) of arm-ECG bipolar leads, simultaneously in a synchronised time window signal levels; after a SFP process SA ECG denoising, and then their comparison with the remaining background noise level, provided an objective quantitative evidence of an expected electrogram activity signal strength distribution along the arm; from the upper arm to the wrist, from Table 1.

For any bipolar arm-ECG lead set data to be of use for cardiac rhythm monitoring the SNR must be at least $>1$ [4], after an adequate ECG denoising process. The distal bipolar leads on the wrist (Lead-2, Lead-6 and Lead-10), presented an average $(\mathrm{N}=11)$ SNR greater than 2. Of practical importance, are the transversally oriented (perpendicular to arm axial line) leads. Of this type, Lead1, Lead-4 and Lead-5, presented highly promising SNR levels above 8 and up to 128 . The later, being recorded with the gelled BIS ${ }^{\mathrm{TM}}$ sensor system.

This pilot clinical study on 11 subjects, revealed an
ECG SNR distribution map on the left arm. Furthermore, as the results evidenced that far-field bipolar arm-ECG signals were in average, consistently stronger on the upper arm, when recorded with the pre-gelled type BISQuatro $^{\mathrm{TM}}$ sensors, it encouraged a larger study for datadriven denoising solutions, using the gelled BIS sensors.

\section{Acknowledgements}

This research is supported by funding from the European Union (EU): H2020-MSCA-RISE Programme (WASTCArD Project, Grant \#645759).

\section{References}

[1] Katritsis DG, Gersh BJ, Camm AJ (2016). A clinical perspective on sudden cardiac death. Arrhythmia and Electrophysiology Review, 2016; 5 (3): 177-182..

[2] Krahn AD, et al. Insertable loop recorder use for detection of intermittent arrhythmias. Pacing and Clinical Electrophysiology, 2004; 27:657-64.

[3] Massot B, Noury N, Gehin C, McAdams ET. On designing an ubiquitous sensor network for health monitoring. IEEE 15th International Conference on e-Health Networking, Applications and Services, Healthcom, 2013; pp. 310-314.

[4] Lynn WD, Escalona OJ, McEneaney DJ. Arm and wrist surface potential mapping for wearable ECG rhythm recording devices: a pilot clinical study. Journal of Physics: Conference Series, 2013; 450, 012026.

[5] Escalona OJ, Mendoza M (2016). "Electrocardiographic Waveforms Fitness Check Device Technique for Sudden Cardiac Death Risk Screening". Proceedings of IEEEEMBS, 2016, Article number 7591471, pp. 3453-3456.

[6] Gemperle F, Kasabach C., Stivoric J., Bauer M., Martin R. Design for Wearability. ISWC Second International Symposium on Wearable Computers (ISWC'98), 1998; 116.

[7] Escalona O J, Mitchell RH, Balderson DE. A fast and reliable QRS alignment technique for high-frequency analysis of the signal-averaged ECG, Medical and Biological Engineering and Computers, 1993; 31: 137-146.

Address for correspondence.

Name: Omar J Escalona

Full postal address: Ulster University, Shore Road, Newtownabbey, BT37 0QB, UK.

E-mail address: oj.escalona@ulster.ac.uk 